In Cres. Vol. $3 N^{\circ}$ 2: pp. 239-246, 2012

\title{
EL USO DE LOS MAPAS CONCEPTUALES COMO ESTRATEGIA COGNITIVA PARA ESTUDIANTES DE MEDICINA DE LA UNIVERSIDAD PERUANA CAYETANO HEREDIA*
}

\author{
THE USE OF CONCEPTUAL MAPS AS A COGNITIVE STRATEGIES \\ FOR MEDICINE STUDENTS AT UNIVERSIDAD \\ PERUANA CAYETANO HEREDIA
}

Emma Margarita Wong Fajardo

\section{RESUMEN}

El artículo presenta resultados de la experiencia del uso de mapas conceptuales como estrategia cognitiva en el curso de Comunicación y Aprendizaje. La población está constituida por estudiantes varones y mujeres que cursan el primer año de estudios en la Facultad de Medicina de la Universidad Peruana Cayetano Heredia; la edad promedio de este grupo es de 16 años. En su mayoría proceden de escuelas privadas y un pequeño porcentaje, de escuelas preuniversitarias y estatales.

La experiencia muestra cómo el uso de los mapas conceptuales complementados con el Cmap tools, aportan al cumplimiento de la competencia general del sílabo, expresada en que los estudiantes "identifiquen, seleccionen y apliquen estrategias de aprendizaje para mejorar su desempeño académico y logren aprender de manera autónoma. La construcción de mapas se inició de forma colaborativa, hasta lograr un afianzamiento por los estudiantes, que les permitiera estar en condiciones de elaborar sus mapas individuales. Para la elaboración personal de los mapas conceptuales los estudiantes seleccionaron temas de otras asignaturas que necesitaban aprender; luego organizaron la información ,estableciendo criterios de selección de conceptos relevantes, para luego representarlos jerárquicamente en los mapas conceptuales definitivos, los mismos que cumplen con los siguientes criterios técnicos: concep-

* Recibido: 04 de mayo del 2012; aprobado: 25 de octubre del 2012.

1 Magister en Docencia e Investigación en Educación Superior Universidad Peruana Cayetano Heredia (2007). Egresada del doctorado en Educación (Universidad San Martín de Porres-2010). Coordinadora de la Maestría en Educación en la FAEDU de la Universidad Peruana Cayetano Heredia y Evaluador Externo de la carrera de Educación acreditado por el CONEAU. 
tos claves, pertinencia de conectores e impacto visual . La organización y presentación final de los mapas ha permitido que los estudiantes almacenen la información en sus propias estructuras cognitivas, la cual manifiestan haber recuperado en los exámenes, convirtiéndose en evidencia, de que los contenidos seleccionados han sido comprendidos.

PALABRAS CLAVE: Mapas conceptuales, Estrategias de aprendizaje, Aprendizaje autónomo.

\section{ABSTRACT}

The article presents the results of using concepual maps as a cognitive strategy in the subject of communication and learning. The population is integrated by 1 st year medicine students of Universidad Peruana Cayetano Heredia. The students' average age was 16. Most of them came from private schools and the rest from public and pre-universitary ones.

This study gives evidence of the benefits by using conceptual maps together with the Cmap tools into the learning process, facilitating the acomplishment of the sylabus. By using them, the students can identify, select and apply learning strategies improving their academic performance as well as their self-learning as a result. At first, the building of maps began as a colaborative way and gradually the students could make it by themselves. The contents of these conceptual maps were the subject topics they needed to learn. They organized and selected the most relevant concepts and finally represented jerarquily in the conceptual maps, which meets the following criteria; key concepts, appropriate connectors and visial impact. The organization and the presentation of the maps have helped students to store information in their own cognitive structures, the ones have been recall at the time of the exam, evidencing the choosen contents had been understood.

KEY WORDS: Conceptual maps, Learning strategies, Autonomous learning.

\section{INTRODUCCIÓN}

Se puede observar por las publicaciones existentes que una de las líneas de investigación que más resultados ha brindado en los últimos años son las relacionadas con las estrategias de aprendizaje, puesto que el tema de mejorar el aprendizaje, así como de los factores que inciden en el mismo, siempre son una preocupación permanente dentro del ámbito de las instituciones educativas de todos los niveles de enseñanza.

En una de sus publicaciones, Monereo C. (1999) indica que el aprendizaje centrado exclusivamente en la adquisición de contenidos específicos, en su mayor parte de tipo declarativo, sin la enseñanza asociada y explícita de estrategias de aprendizaje conduce a un conocimiento inerte que no puede emplearse de manera funcional. Agrega el autor; “...la utilización de estrategias, al requerir una toma consciente de decisiones, adaptadas a las condiciones de cada situación, y orientadas a unos objetivos, hacen que esos conocimientos resulten accesibles y, por lo tanto, útiles". 
Para autores, como Cfr. N. J. y Marton, F, (1991), citado por Valle,A.(1998), las investigaciones sobre las estrategias de aprendizaje junto con la teoría del procesamiento de la información constituyen las aportaciones más relevantes de la psicología cognitiva al estudio del aprendizaje escolar"; así mismo destacan que "la importancia de las estrategias de aprendizaje viene dada por el hecho de que engloban aquellos recursos cognitivos que utiliza el estudiante cuando se enfrenta al aprendizaje; por lo tanto, el referirse a las estrategias de aprendizaje suele ser sinónimo de "cómo aprender".

Una estrategia de aprendizaje es un procedimiento; es decir, un conjunto de pasos o habilidades que un alumno adquiere y emplea en forma intencional como instrumento flexible para aprender significativamente, solucionar problemas y demandas académicas; además, conseguir una meta propuesta. Estas son ejecutadas voluntaria, flexible e intencionalmente por un aprendiz (Díaz y Hernández, 1998).

El mapa conceptual es una estrategia de aprendizaje de tipo cognitivo, que permite exteriorizar la estructura cognitiva de una persona en forma clara y organizada. Joseph Novak, creador de los mapas conceptuales, fue quien inició sus experiencias con los estudiantes de la Universidad de Cornell (USA) entre los años 1975-1977 y, posteriormente, con alumnos de primaria (1981) y secundaria (1983).

Novak parte de la idea de Ausubel, según la cual los conceptos y principios son los bloques de construcción del conocimiento, las herramientas que nos permiten pensar; por eso, el mejor modo de ayudar a los estudiante a aprender significativamente es ayudando a que identifiquen los conceptos y las relaciones entre ellos, tal como existen en sus mentes y como existen fuera, en la realidad, o en el mundo del conocimiento especializado (Novak y Gowin, 1988).

En la experiencia que presentamos, se recoge los aportes teóricos de los autores citados, se refiere cómo los estudiantes de la facultad de medicina de una universidad peruana, identifican, aprenden, elaboran y potencian el uso de los mapas conceptuales para estudiar temas de las diversas asignaturas que forman parte de su plan de estudios. La secuencia de estos procesos se desarrolla al interior de la asignatura de Comunicación y Aprendizaje, que es de corte propedéutico y se desarrolla en el primer año de la carrera mencionada.

Los principios técnicos para el aprendizaje de los mapas conceptuales fueron desarrollados por las docentes encargadas de los diferentes grupos de estudiantes (4 en total) y la elaboración de los mismos se desarrolló, primero, de 
manera individual y luego, colaborativa. Después de verificada la correcta construcción de los mapas, en una etapa posterior, se enseñó el uso correcto del Cmap tools, a fin de potenciar los trabajos elaborados de manera personal, con temáticas propias de las asignaturas que forman el plan de estudios del primer año de la carrera de medicina.

\section{EXPERIENCIA}

La Facultad de Medicina de la Universidad Peruana Cayetano Heredia, tiene identificadas en el currículo, aquellas competencias que el egresado debe alcanzar a lo largo de su formación profesional y ha seleccionado asignaturas que aportan a cada una de ellas. En el plan de estudios de esta carrera, se identifica en el listado de asignaturas a Comunicación y Aprendizaje, que forma parte de la presente experiencia.

En la sumilla del curso Comunicación y Aprendizaje se destaca que los estudiantes desarrollen habilidades para aprender de manera autónoma manejando estrategias para el estudio; a partir de esta sumilla se considera, entre las competencias del sílabo, que el estudiante: identifique, seleccione y aplique estrategias de aprendizaje para mejorar su desempeño académico y aprender de manera autónoma. Es así que el sílabo incluye y destaca entre los contenidos a desarrollar a las estrategias de aprendizaje, y los mapas conceptuales se seleccionan como una de estas estrategias.

Ontoria, Molina y Luque (1996) sostienen que los mapas conceptuales trabajan cuatro aspectos básicos sobre el aprendizaje significativo: la conexión con los conocimientos previos, la inclusión, la diferenciación progresiva y la reconciliación integradora; reconocemos como muy importantes desde nuestra perspectiva docente que los estudiantes utilicen para el aprendizaje de asignaturas de su plan estudios los mapas conceptuales como una estrategia que ayude a organizar y aprender los contenidos deseados.

Debido a la potencialidad de los mapas conceptuales como herramienta conceptual y que esta formulación se respalda en todas las investigaciones que se reportan en eventos, tesis, publicaciones, decidimos afrontar la tarea de utilizar los mapas como una estrategia de aprendizaje cuya secuencia de intervención se detalla de la siguiente manera:

En la primera intervención, se preguntó a los alumnos sobre las experiencias previas que habían tenido con el uso de mapas conceptuales. La mayoría refirió que sí habían tenido estas experiencias previas y que sabían elaborar 
mapas. Entonces, se les proporcionó una lectura titulada "Condiciones del Aprendizaje”, cuyo autor es Aníbal Meza, y se les pidió que elaboren una mapa individual; acto seguido, se agrupó al azar en número de seis estudiantes y se les pidió que elaboren un mapa grupal del mismo tema, el cual fue mostrado mediante la técnica del museo.

En la segunda intervención, el docente explicó la teoría sobre el origen de los mapas conceptuales, sus usos, la correcta construcción y los criterios de evaluación. Con esta información, el docente procedió a corregir los mapas grupales, aplicando los criterios técnicos que se habían mostrado en la explicación teórica. Esta segunda etapa sugirió una serie de preguntas, cuestionamientos por parte de los estudiantes, puesto que los mapas presentaban una serie de errores y se priorizó, en las respuestas docentes, que la metodología, teoría y uso de los mapas, quede consolidada como aprendizaje teórico y práctico.

La tercera intervención consideró que los estudiantes con los aprendizajes logrados, elaboren mapas conceptuales de manera individual. El tema sería de la asignatura que ellos eligiesen y que era muy importante que aprendan. Se les informó sobre la existencia del software Cmap tools y sobre las experiencias en diversos congresos, en los que se hace uso de los mapas con el software mencionando. Por exploración los estudiantes usaron el Cmap tools para elaborar sus mapas, los cuales fueron corregidos de acuerdo a los criterios establecidos en clase.

Finalmente, en la última intervención, los estudiantes recibieron una capacitación sobre el uso del Cmap tools en los laboratorios de cómputo de la universidad, para potenciar la utilización de la herramienta y se dejó la posibilidad que presenten el mapa inicial mejorado, incorporando ayudas multimediales que el software les ofrece.

La experiencia del uso de mapas conceptuales con los estudiantes ha generado la aceptación y el gusto por esta estrategia, teniendo en cuenta, en un principio, el desagrado como herramienta de estudio que traían de la etapa escolar. Después de la experiencia, la selección de dicha estrategia, especialmente para cursos que los estudiantes consideran "difíciles" por su complejidad, vocabulario denso y abundante información, como: matemática, química, ecología, primeros auxilios (a decir de los propios estudiantes); además de lo atractivo que resulta para el estudiante la aplicación mediante el Cmap tools, en esta era del aprendiz tecnológico en la cual se encuentran inmersos nuestros estudiantes. 


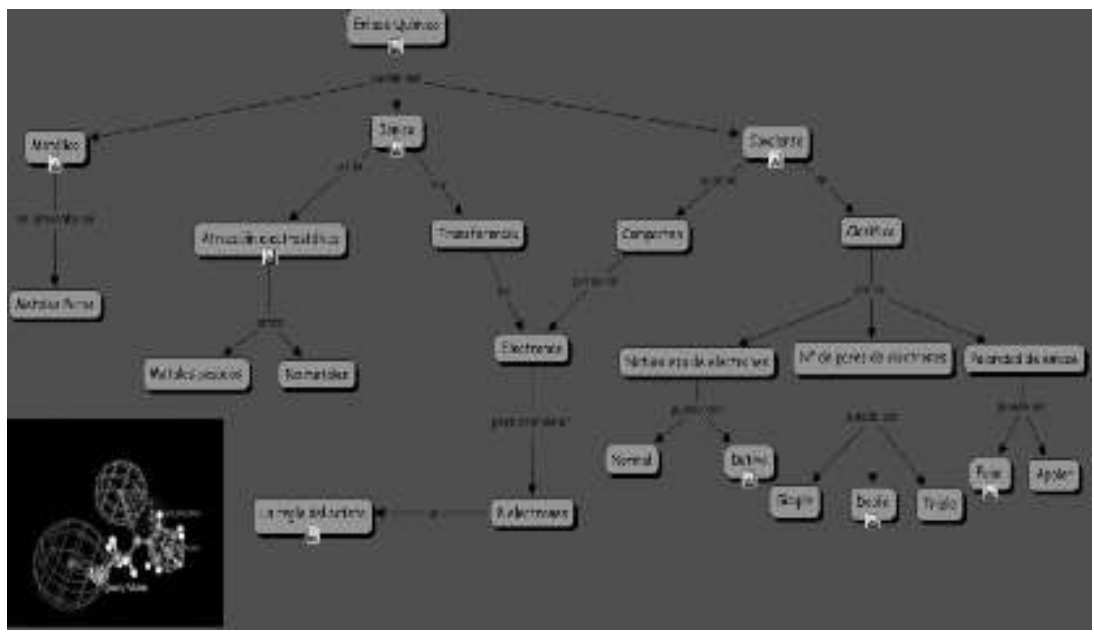

Figura 1. Mapa conceptual asignatura: Química.

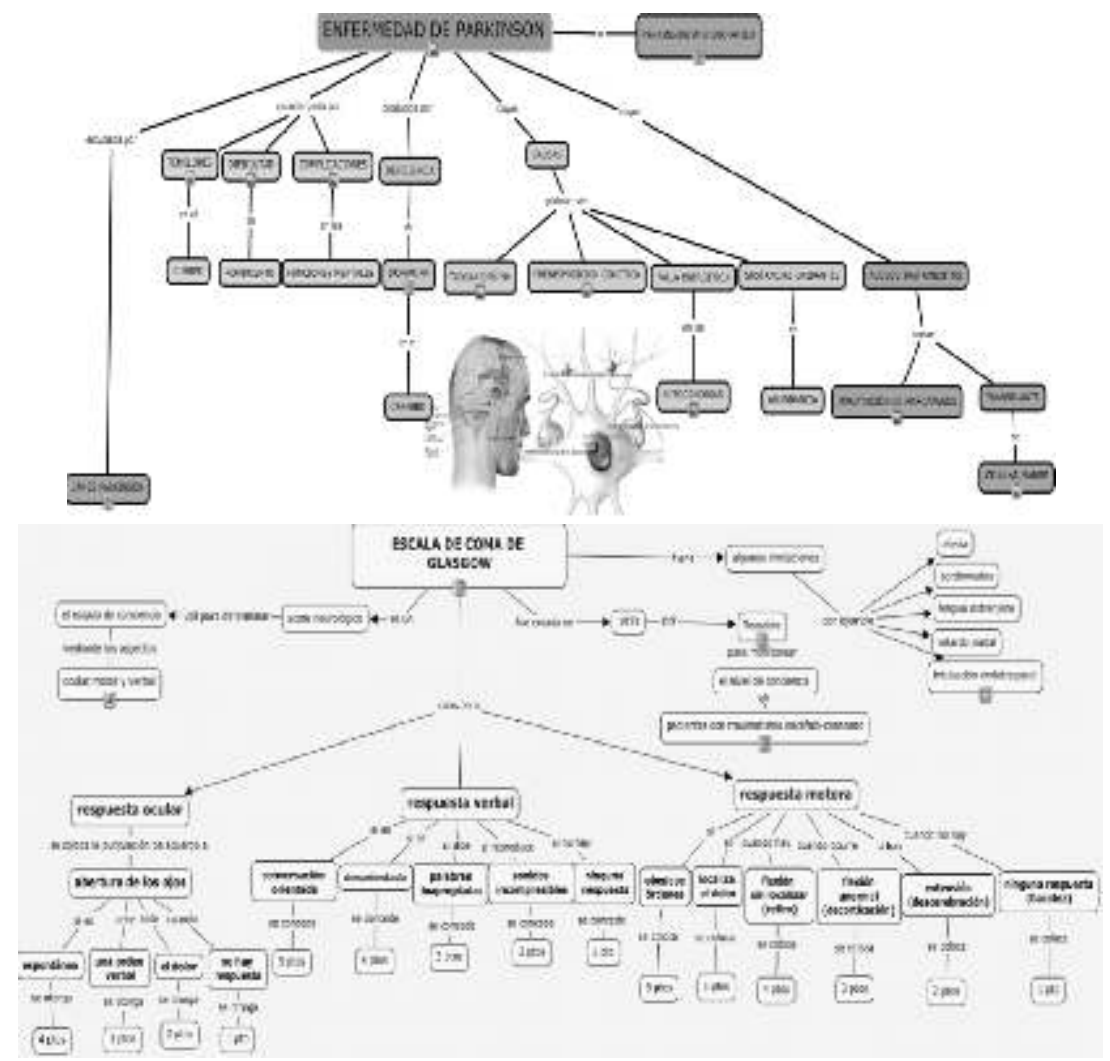

Figura 2. Mapa conceptual asignatura: Primeros auxilios. 
El uso de los mapas conceptuales como estrategia cognitiva...

\section{RESUMEN}

Desde sus inicios la educación médica exige un abordaje de temas muy complejos, correspondiéndole a los profesores implementar nuevas estrategias que ayuden al estudiante a generar conocimientos que se perciban significativos durante su preparación profesional.

El trabajo muestra la experiencia realizada por los alumnos de la Facultad de Medicina de la Universidad Peruana Cayetano Heredia, en tres momentos de aprendizaje, utilizando los mapas conceptuales mediante un software especializado (Cmap tools). Los resultados más evidentes, a decir de los estudiantes, les ha permitido desarrollar autonomía, así como construir sus propias rutas cognitivas para aprender de manera significativa contenidos de otros cursos del plan de estudios. La organización y presentación final de los mapas conceptuales, ha permitido a los estudiantes almacenar conocimientos de las diversas temáticas en sus propias estructuras cognitivas, manifestando haber mejorado su desempeño académico.

\section{CONCLUSIONES}

1. Es necesario que los docentes implementen nuevas estrategias que ayuden al estudiante a generar conocimientos que los consideren útiles y significativos, y de esta manera evitar que sean simples receptores de información.

2. Los estudiantes destacaron la utilidad de los mapas conceptuales para aprender temas de los cursos que ellos manifestaban muy difíciles, por la densa información, su complejidad, y vocabulario técnico.

3. El Sofware Cmap tools resultó muy atractivo para los estudiantes, y ha permitido utilizar las herramientas propias del aprendiz tecnológico.

4. En el trabajo se aprecia que el uso de los mapas conceptuales ha permitido exteriorizar la estructura cognitiva de cada estudiante; los mapas elaborados se aprecian claros y organizados.

\section{BIBLIOGRAFÍA}

Ontoria, A., Rubio, A. y LuQue, A. (1996). Los mapas conceptuales en el aula. Buenos Aires: Ed. Magisterio de La Plata.

MONEREO, C.(1999) Estrategias de enseñanza Aprendizaje :Formación del profesorado y aplicación en la escuela. Disponible en :http://www.terras.edu.ar/jornadas/119/biblio/79Las-estrategias-deaprendizaje.pdf. Fecha de consulta: 23.04.12. 
Cfr. N. J. y MARTON, F. (1998). Knowledge objects: Understandings constituted trhrough intensive academic study. British Journal of Educational Psychology, 64 (1991), 161-178. En Valle, A y otros (1998).Disponible en: http://redalyc.uaemex.mx/redalyc/pdf/175/17514484006.pdf. Fecha de consulta: 20.04.2012.

DíAz, F. y Hernández, G. (1998). Estrategias Docentes para un Aprendizaje Significativo. Una interpretación constructivista. México: Ed. McGraw Hill.

NovaK y Gowin (1988). Aprendiendo a Aprender. Barcelona, Martínez Roca.

MEZA, A. (1998). Para conocer la Psicología. Lima, Perú.

CAÑAS y otros. Colaboración en la Construcción de Conocimiento Mediante Mapas Conceptuales. http://www.ihmc.us/users/acanas/ColabCon.pdf. Fecha de consulta: 11.07.2012.

CAÑAS, A. Volúmenes de memorias de los Cinco Congresos Internacionales de Mapas Conceptuales. http://cmc.ihmc.us/cmc/CMCProceedings.html. Setiembre 2012. 